\title{
Krüppel-Like Factor 5 Promotes Lung Tumorigenesis through Upregulation of Sox4
}

\author{
Qi Lij ${ }^{\mathrm{a}, \mathrm{d}}$ Zhao Dong ${ }^{\mathrm{b}, \mathrm{d}}$ Fei Zhou ${ }^{\mathrm{a}}$ Xun Cai ${ }^{\mathrm{a}}$ Yong Gao ${ }^{\mathrm{c}}$ Li-Wei Wang ${ }^{\mathrm{a}}$ \\ aDepartment of Oncology, Shanghai First People's Hospital Affiliated Shanghai Jiao Tong University, \\ bDepartment of Radiotherapy, No.85 Hospital of People's Liberation Army, 'Department of Oncology, \\ Shanghai East Hospital, Tong Ji University, Shanghai, China; ${ }^{\mathrm{d}}$ These people contributed equally
}

\author{
Key Words \\ KLF5 • Sox4 • Lung cancer $•$ Cell proliferation
}

\begin{abstract}
Background: Krüppel-like factor 5 (KLF5), a member of zinc finger class of DNA-binding transcriptional regulators, has attracted attention because of its important regulatory activities linked to diverse functions such as cell growth, proliferation, differentiation, apoptosis and tumorigenesis in a number of systems. However, its biological functions in the initiation and progression of lung tumorigenesis remain largely unexplored. Methods: Quantitative realtime PCR and Western Blot were used to detect the expression of KLF5 in lung cancer tissues and cell lines. Retro-viruses were used to generate KLF5 stable expression lung cancer cell line. Small interfering RNA was used to silence the expression of KLF5 and Sox4. BrdU assay was used to determine the proliferation of cells. Luciferase and Chromatin immunoprecipitation assays were used to detect the regulation of Sox4 by KLF5. Results: KLF5 was up-regulated in lung cancer tissues and cell lines. Overexpression of KLF5 promotes while knockdown of its expression inhibits cell proliferation in two cell lines derived from lung carcinoma. At the molecular level, our results revealed that KLF5 positively regulates Sox4 expression through a transcriptional mechanism. Sox4 deficiency blocked the proliferative roles of KLF5 in lung cancer cells. Conclusion: our data identified the KLF5/Sox4 regulatory signaling play an important role in lung tumorigenesis, which might represent novel therapeutic targets to manage lung carcinoma.
\end{abstract}


Li et al.: KLF5 Promotes Lung Tumorigenesis

\section{Introduction}

Lung cancer is the leading cause of cancer-related mortality with global statistics indicating nearly 1.4 million deaths annually $[1,2]$. Although advances have been obtained in systemic therapy in conjunction with local therapy, the prognosis after treatment still remains poor $[3,4]$. Therefore, it is important to get a better understanding of the molecular mechanisms of lung cancer progression in order to develop more effective therapies.

Members of the Krüppel-like factor (KLF) are transcription factors containing a zinc finger domain [5]. KLF proteins were originally identified in Drosophila melanogaster, in which they play essential roles in embryogenesis [6]. In mammals they constitute a large family of factors containing 17 members that bind to a CACCC or CGCCC DNA motif $[7,8]$. Some of these transcription factors act as transcriptional activators while some act as transcriptional repressors and other members have a dual role in both activating and repressing gene expression [9]. They have a wide range of roles in the regulation of development, cell cycle, differentiation and apoptosis [7, 8, 10, 11]. Recent studies also suggest a potential function of KLFs in tumor progression [12]. Expression of KLF4, for instance, was decreased or lost in primary tumors and, in particular, lymph node metastases when compared with that in normal gastric mucosa [13]. KLF4 also inhibits the growth and metastasis of human pancreatic cancer by transactivating p27 (Kip1) expression [14]. On the other hand, KLF8 is highly overexpressed in several types of human cancers and regulates various cellular processes important for tumor progression [15].

KLF5 was initially identified as a positive regulator of SMemb, a marker gene for activated smooth muscle cells in vascular disease [16]. Subsequent studies revealed that KLF5 mediated cardiovascular remodeling and adipogenesis in vivo $[17,18]$. In tumors, levels of KLF5 expression increased in parallel with advancing stages of neoplastic progression in patients with gastric cancer [19]. Besides, up-regulation of Krüppel-like factor 5 was also observed in pancreatic cancer due to interleukin-1beta signaling and hypoxia-inducible factor-1alpha [20]. However, its roles in lung carcinoma remain unexplored. Therefore, we will investigate its expression and functions in the present study.

\section{Materials and Methods}

Tissue samples

Primary lung carcinoma tissues, adjacent normal lung tissues were collected from routine therapeutic surgery at our department. All samples were obtained with informed consent and approved by Shanghai First People's Hospital Affiliated Shanghai Jiao Tong University.

Cell culture

All cell lines used were purchased from The Cell Bank of Type Culture Collection of Chinese Academy of Sciences (CAS, Shanghai), and cultured in Dulbecco modified Eagle's medium supplemented with 10\% fetal calf serum, $100 \mathrm{IU} / \mathrm{ml}$ penicillin and $100 \mathrm{mg} / \mathrm{ml}$ streptomycin.

\section{Transient Transfections and Luciferase assays}

Human Sox4 promoter was amplified from the human genomic DNA template and inserted into pGL3 basic vector (Promega). Mutant KLF5 binding motif was generated using a PCR mutagenesis kit (Toyobo). All the transient transfections were performed by Lipofectamine 2000 (Invitrogen), according to the manufacturer's instructions. For luciferase reporter assays, A549 cells were seeded in 24-well plates and transfected with the indicated plasmids. Cells were harvested $24 \mathrm{hr}$ after transfection. Luciferase activities were measured using the Dual Luciferase Reporter Assay System (Promega, USA).

siRNA, RNA extraction and Real-time Analysis

Cells were seeded on to 6-well plates then transfected with 25nM siGENOME non-targeting siRNA, human KLF5 or Sox4 (Dharmacon, USA). Total RNAs were isolated from tissues or cells by TRIzol reagent, and 
Fig. 1. Up-regulation of KLF5 in lung cancer tissues and cell lines. (A-B) mRNA (A) and representative protein (B) levels of KLF5 were analyzed by real-time PCR and western blot in lung cancer tissues or normal tissues. C: lung cancer tissues; N: normal tissues. (C-D) mRNA (C) and protein (D) levels of KLF5 were analyzed in normal lung epithelia cells (MRC-9 cells) and several lung cancer cell lines (A549, Calu-3 and NCI-H292).

\section{A}

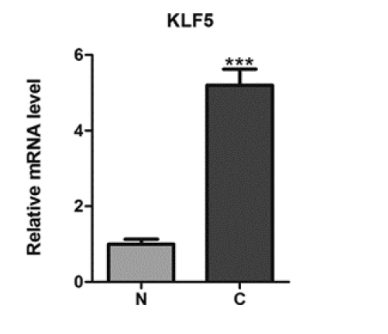

C

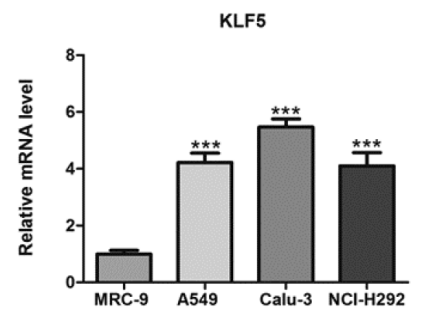

B

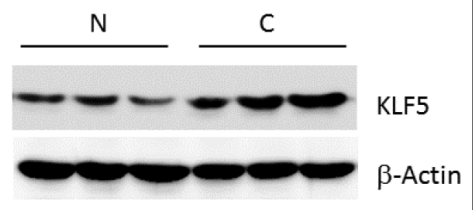

MRC-9 A549 Calu-3 NCI-H292

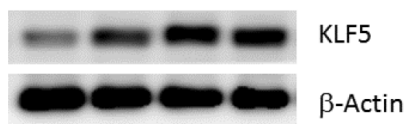

reverse transcriptions were performed by Takara RNA PCR kit (Takara, China) following the manufacturer's protocols. In order to quantify the transcripts of the interest genes, real-time PCR was performed using a SYBR Green Premix Ex Taq (Takara, Japan) on Light Cycler 480 (Roche, Switzerland).

\section{Western Blot}

Cells or tissues were lysed by trypsinization, lysed in Laemmli buffer $(100 \mathrm{mM}$ Tris- $\mathrm{HCl}$ at $\mathrm{pH} 6.8$, $200 \mathrm{mM} \mathrm{DTT}, 4 \%$ SDS [w/v], 20\% glycerol, $0.05 \%$ bromophenol blue), denatured for $10 \mathrm{~min}$ at $80^{\circ} \mathrm{C}$, sheared with an insulin syringe, and resolved on SDS/PAGE gels. After immunoblotting, the membranes were blocked in PBS/0.1\% Tween-20 with 5\% nonfat dry milk, and primary antibodies were incubated in PBS $/ 0.1 \%$ Tween-20 with 0.1\%-5\% nonfat dry milk. Antibodies directed against KLF5, Sox4 and $\beta$-actin were purchased from Santa Cruz Biotechnology (USA). Anti-Cyclin B1, Cyclin D1, p27 and p21 were obtained from Abcam Company (USA).

ChIP Assays

Chromatin immunoprecipitation (ChIP) assay kits were used (Upstate, USA). In short, A549 cells were fixed with $1 \%$ formaldehyde to cross-link the proteins and DNA, followed by sonication in an ultrasound bath on ice. DNA was sheared to fragments at 200-1000 bp using sonication. The chromatin was then incubated and precipitated with the KLF5 antibody or IgG (Abcam, USA). The immunoprecipitated DNA fragments were detected using real-time PCR analysis.

Statistical Analysis

All data are presented as mean \pm SEM. Statistical differences were determined by a two-tailed test or One Way ANOVA analysis. Statistical significance is displayed as $*(\mathrm{P}<0.05),{ }^{* *}(\mathrm{P}<0.01)$ or *** $(\mathrm{P}<0.001)$.

\section{Results}

Up-regulation of KLF5 is a frequent event in lung cancer tissues or cells.

First, we analyzed KLF5 expression in 25 paired lung cancer and adjacent non-tumor tissues by way of real-time PCR. We found that KLF5 was significantly up-regulated in cancer tissues (Fig. 1A). We then employed western blot with anti-KLF5 antibody to detect the protein levels of KLF5 in those clinic samples, which revealed the increased expression of KLF5 in representative lung cancer samples (Fig. 1B and data not shown). Besides, we 


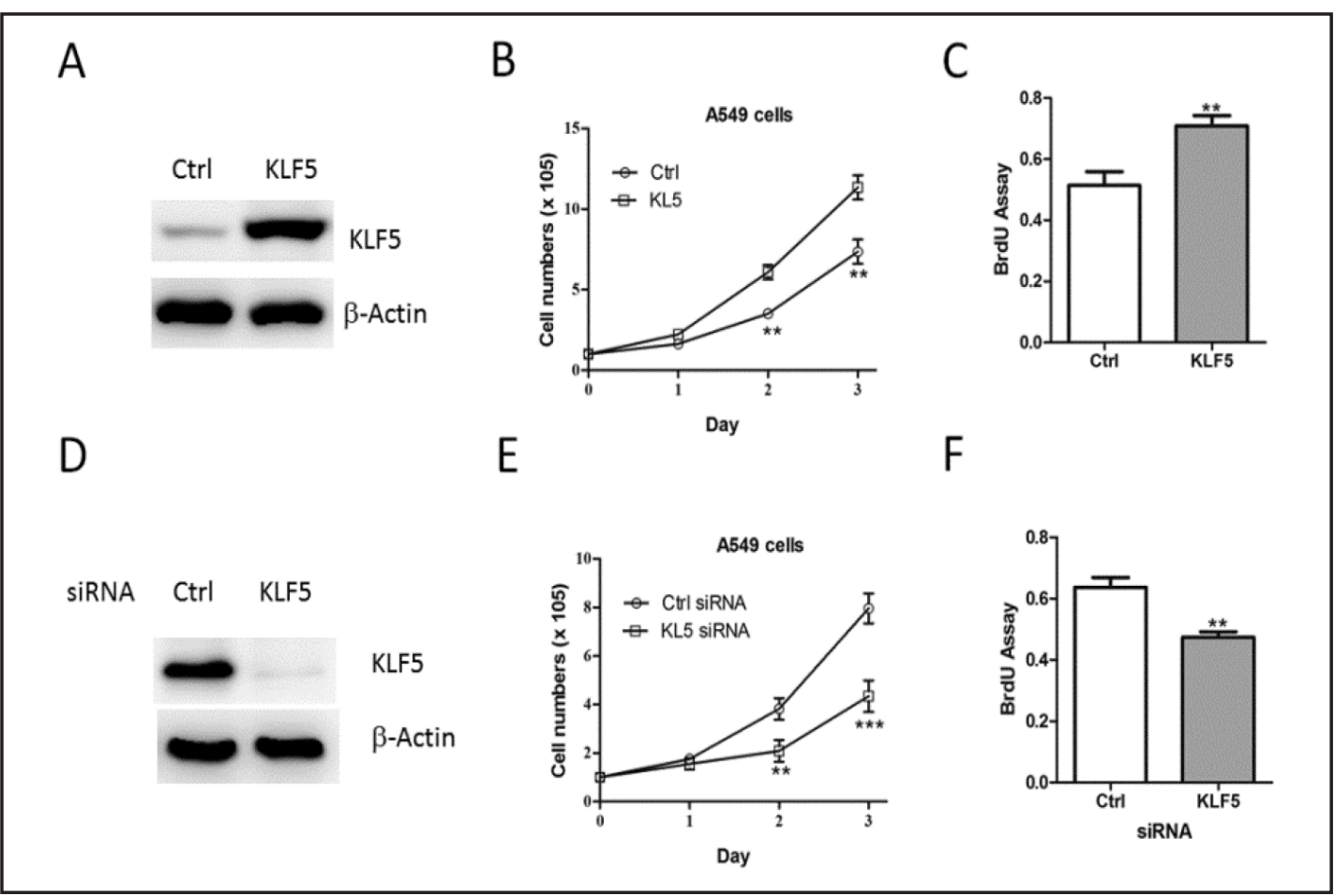

Fig. 2. KLF5 regulates cell proliferation in A549 cells. (A) Protein level of KLF5 was determined by western blot in A549 cells. Cells were transfected with retro-viruses containing empty vector (Ctrl) or KLF5 for $48 \mathrm{hr}$ before harvest. (B) The growth curve of A549 cells transfected with empty vector (Ctrl) or KLF5. (C) The cell proliferative potential (BrdU) was determined in A549 cells transfected with empty vector (Ctrl) or KLF5. (D) Protein level of KLF5 was n was determined by western blot in A549 cells. Cells were transfected with siRNA oligos targeting KLF5 or scramble siRNA (Ctrl) for $48 \mathrm{hr}$ before harvest. (E) The growth curve of A549 cells transfected with transfected with siRNA oligos targeting KLF5 or scramble siRNA (Ctrl). (F) The cell proliferative potential (BrdU) was determined in A549 cells transfected with KLF5 or scramble siRNA (Ctrl).

also observed a higher expression of KLF5 in three lung cancer cell lines (A549, Calu-3 and NCI-H292), compared with normal lung epithelia cells (MRC-9) (Fig. 1C and 1D), suggesting that KLF5 expression was up-regulated in lung carcinoma tissues and cell lines.

\section{Effects of KLF5 on lung cancer cell proliferation}

To further elucidate the functional roles of KLF5 in lung tumorigenesis, we transduced A549 cells with retro-viruses containing KLF5 (Fig. 2A). KLF5 overexpression resulted in a significant increase in cell number (Fig. 2B). Consistently, bromodeoxyuridine (BrdU) analysis also confirmed that KLF5 overexpression promoted cell proliferation (Fig. 2C). Next, A549 cells were transfected with small interfering RNA (siRNA) targeting KLF5, which exhibited an efficient KLF5 knockdown in A549 cells, compared with scramble siRNAtransduced cells (Fig. 2D). As a result, down-regulation of KLF5 led to a marked decrease in cell number and proliferation in these cells (Fig. 2E and 2F). Moreover, similar results were also observed in Calu-3 cells (data not shown). Taken together, our results suggest that KLF5 might be an important positive regulator in cell proliferation.

\section{KLF5 affects expression of the cell-cycle regulators}

As KLF5 promoted cell proliferation, we examined its functions on expression of the genes which regulate the cell-cycle transition, such as p21, p27, Cyclin B1 and Cyclin D1. Results from real-time PCR and western blot analysis suggested that expression of p21 and p27 were downregulated while Cyclin B1 and Cyclin D1 levels were upregulated in A549 cells overexpressing KLF5, compared to empty vector (Fig.3A-3B). Similar results were also 
Fig. 3. KLF5 modulates the expression of cell-cycle regulators in A549 cells. (A-B) mRNA (A) and protein (B) levels of p21, p27, Cyclin B1 and Cyclin D1 were determined by real-time PCR and western blot in A549 cells transfected with empty vector (Ctrl) or KLF5. (C-D) mRNA (C) and protein (D) levels of p21, p27, Cyclin B1 and Cyclin D1 were determined by real-time PCR and western blot in A549 cells transfected with siRNA oligos targeting KLF5 or scramble siRNA (Ctrl).

Fig. 4. KLF5 regulates Sox4 expression in A549 cells (A-B) mRNA (A) and protein (B) levels of Sox4, Stat3, Stat5, Fox01, p53 and Rb were determined by real-time PCR and western blot in A549 cells. Cells were transfected with retro-viruses expressing empty vector (Ctrl) or KLF5 for 24 or $48 \mathrm{hr}$, respectively. (C-D) mRNA (C) and protein (D) levels of Sox 4 were determined by real-time PCR and western blot in A549 cells. Cells were transfected with siRNA oligos targeting KLF5 or scramble siRNA (Ctrl) for 24 or 48 $\mathrm{hr}$, respectively.
A

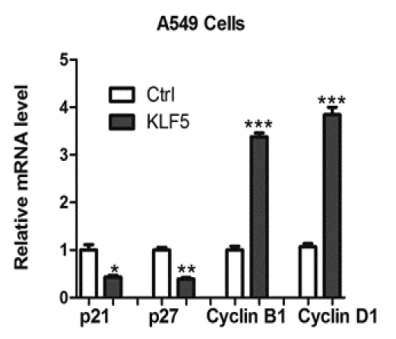

C

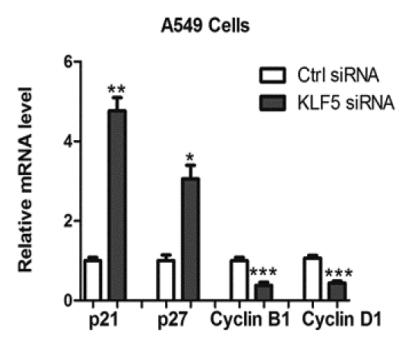

B

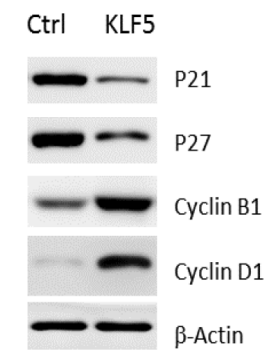

D

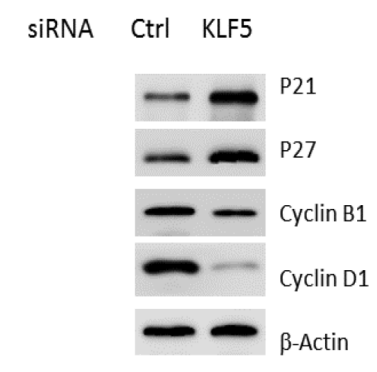

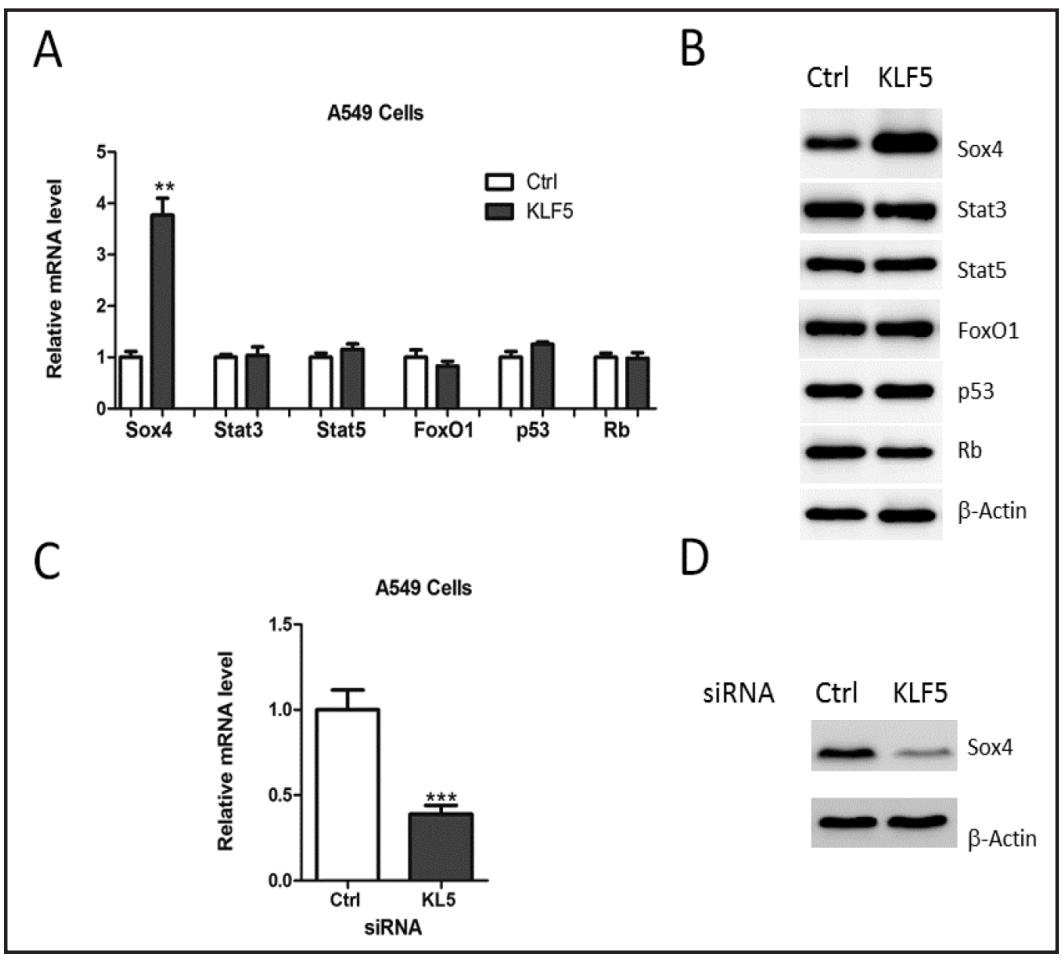

observed in Calu-3 cell (data not shown), further confirming that KLF5 can influence the proliferation of lung cancer cells. Consistently, knockdown of KLF5 increased p21 and p27 expression while reduced Cyclin B1 and Cyclin D1 expression in A549 and Calu-3 cells (Fig. 3C-3D, data not shown).

KLF5 directly targets the transcription factor Sox 4 in lung cancer cells

Previous studies have revealed that numbers of transcriptional factors could regulate expression of genes relevant to the cell-cycle. Thus, we analyzed the potential transcriptional 


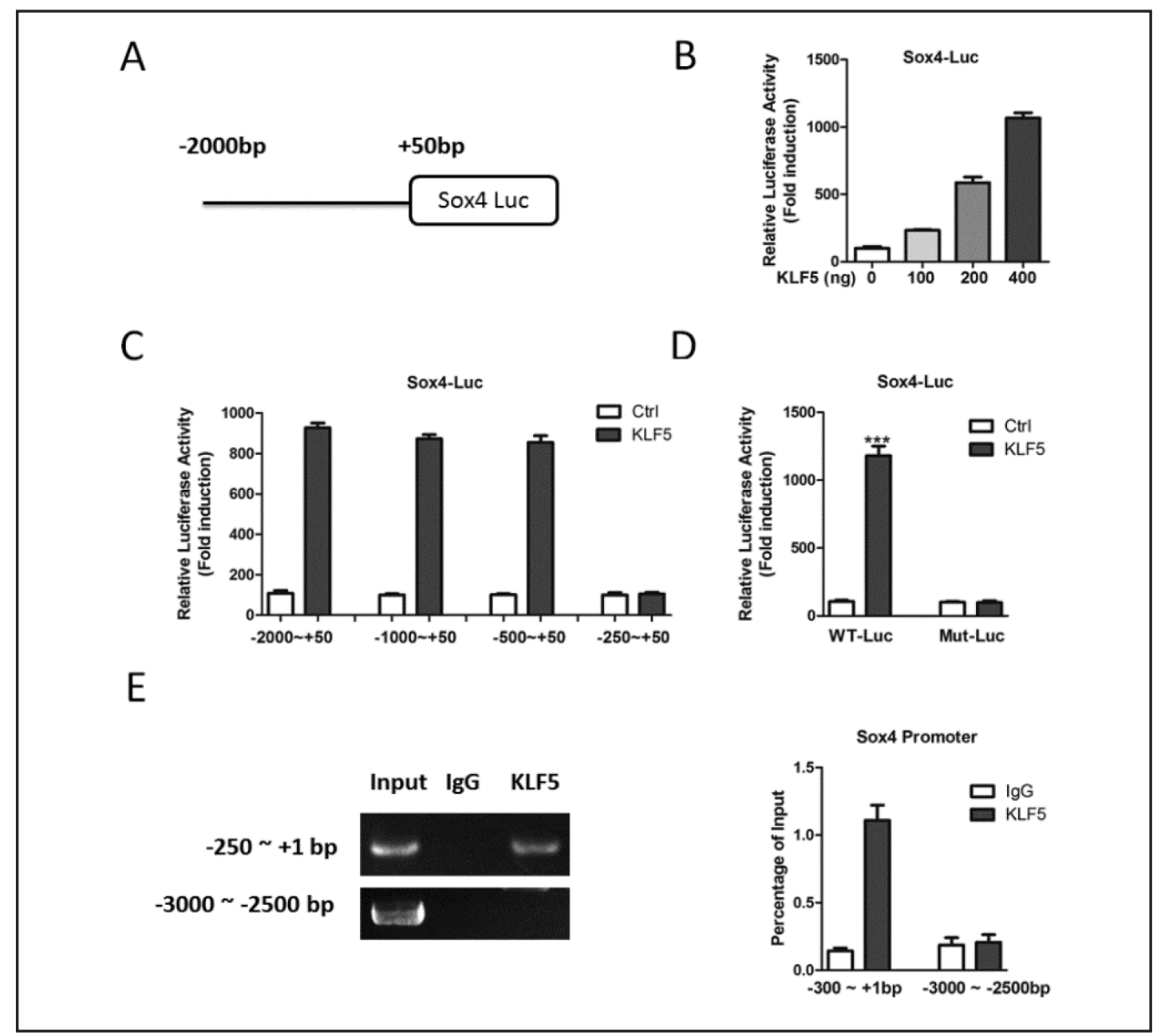

Fig. 5. KLF5 directly up-regulates Sox4 promoter activity. (A-B) Construction and luciferase reporter analysis of human Sox 4 promoter. The promoter region from -2000 to $+50 \mathrm{bp}$ was cloned and co-transfected with KLF5 expression plasmids in A549 cells. The transcription start site was set as +1 bp. (C) Transcriptional activity of human Sox4 promoter by series of deletion. (D) Activation of human wild-type (WT) and mutant (Mut) Sox4 promoter by KLF5. (E) Chromatin immunoprecipitation assays showing the recruitment of KLF5 onto the Sox 4 promoter. The promoter region from -3000 to -2500 bp was set as a negative control. Real-time PCR was performed to quantify the binding activity.

factors that participate in the roles of KLF5. Out of tested transcriptional factors, only Sox 4 were found to be significantly increased in A549 and Calu-3 cells overexpressing KLF5 (Fig. 4A-4B, Supplementary Fig. 4A-4B). In addition, Sox4 expression was reduced in these cells depleted of KLF5 (Fig. 6A-6D, data not shown), suggesting that Sox 4 might be a transcriptional target of KLF5.

The human Sox4 gene promoter is a transcriptional target of KLF5

Next, to further understand the molecular mechanism of this regulation in an independent setting, we examined the activities of reporters driven by Sox 4 gene promoter in A549 cells (Fig. 5A). In accordance with the regulation of the Sox 4 expression, KLF5 activated human Sox 4 promoter activity in a dose dependent manner (Fig. 5B). Through serials deletion of this promoter, we defined a minimal KLF5-responsive region (287 to 283 bp upstream of the transcriptional start site) that contained a consensus binding site for KLF5 (5'-CACCC-3') (Fig. 5C). Besides, mutation of this site completely abolished the effect of KLF5 on Sox4 


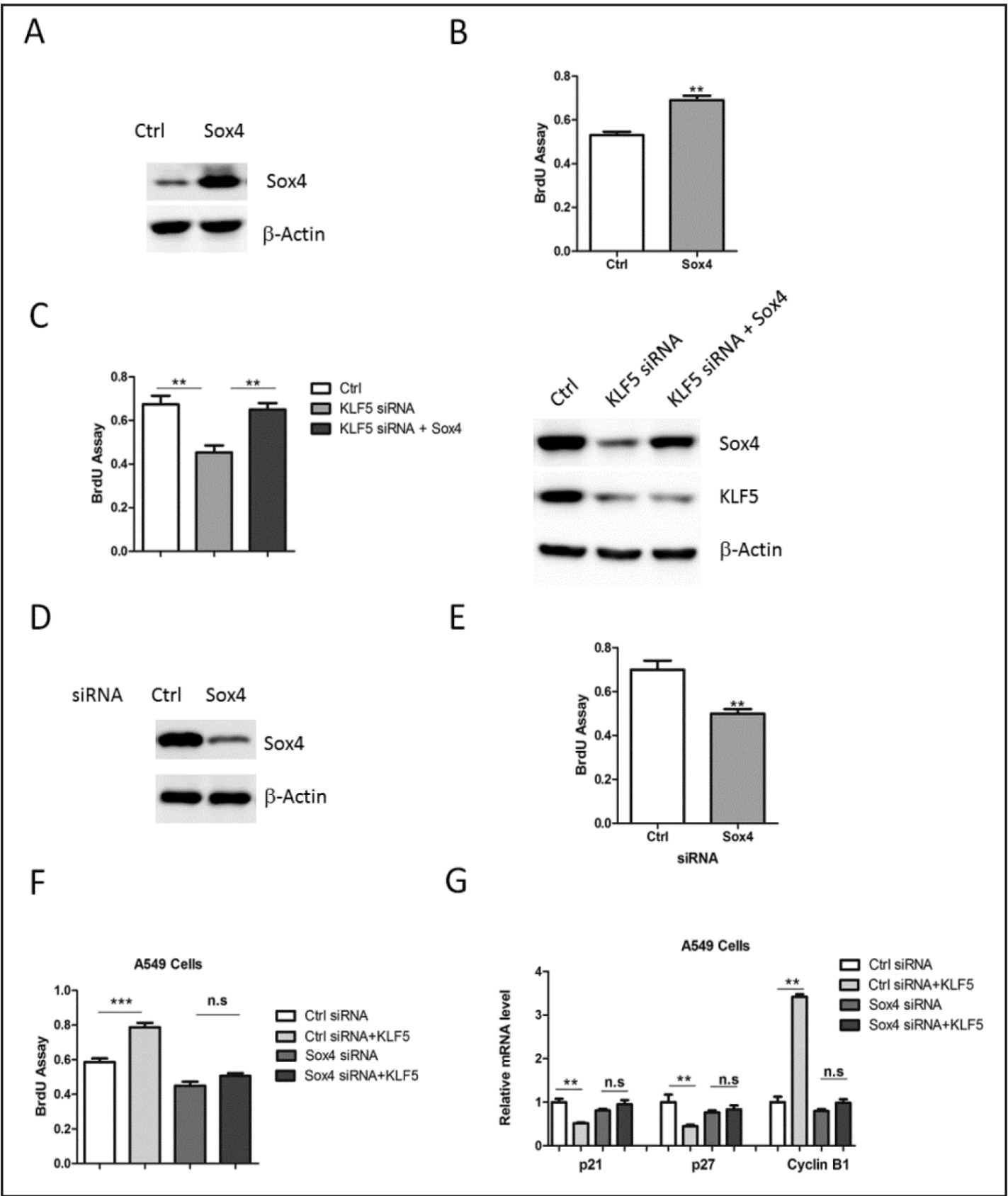

Fig. 6. The proliferative action of KLF5 relies on its up-regulation of Sox4. (A) Protein level of Sox4 expression was determined by western blot in A549 cells. Cells were transfected with retro-viruses containing empty vector (Ctrl) or Sox 4 for $48 \mathrm{hr}$. (B) The cell proliferative potential (BrdU) analysis in A549 cells transfected with empty vector (Ctrl) or Sox4. (C) The cell proliferative potential (BrdU) analysis in A549 cells. Cells were pre-transfected with siRNA oligos against KLF5 or scramble siRNA (Ctrl) for 24 hours. Then cells were transfected with empty vector or Sox 4 for another 24 hours. Results from western blot showing the protein levels of Sox4 and KLF5 in A549 cells. (D) Protein level of Sox4 was determined by western blot in A549 cells. Cells were transfected with siRNA oligos targeting Sox 4 or scramble siRNA (Ctrl) for 48 hr. (E) The cell proliferative potential (BrdU) analysis in A549 cells transfected with siRNA oligos targeting Sox4 or scramble siRNA (Ctrl). (F) Cell proliferation activity was measured by BrdU incorporation assays in A549 cells. Cells were pre-transfected with siRNA oligos against Sox 4 or scramble siRNA (Ctrl) for 24 hours. Then cells were transfected with empty vector or KLF5 for another 24 hours. (G) mRNA levels of p21, p27 and Cyclin B1 were determined by real-time PCR in A549 cells. 
Li et al.: KLF5 Promotes Lung Tumorigenesis

transcriptional activity, confirming that this site was required for the induction of promoter activities by KLF5 (Fig. 5D). In addition, our chromatin immunoprecipitation (ChIP) assays also indicated that KLF5 was able to bind with this region of the Sox4 promoter in A549 cells (Fig. 5E).

\section{Sox4 is required for the KLF5-mediated cell proliferation}

Having established Sox4 as a KLF5 target gene, we went on to determine whether the expression of Sox 4 was required for the proliferative effect of KFL5. BrdU analysis found that Sox4 overexpression promoted cell proliferation in A549 cells (Fig. 6A-6B). Besides, Sox 4 re-introduction largely reversed the proliferation activity of cells inhibited by KLF5 siRNA (Fig. 6C). Then, knockdown of endogenous Sox4 expression was employed using its specific siRNA oligos (Fig. 6D). As a result, Sox4 depletion resulted in a reduction of cell proliferation (Fig. 6E). Moreover, we observed that KLF5 overexpression could not regulate cell proliferation and expression levels of cell-cycle regulators in cells with Sox 4 depletion (Fig. 6F-6G). Together, our results suggest that the proliferative effect of KLF5 in lung cancer is, at least in part, dependent on its induction of Sox4 levels.

\section{Discussion}

The development and progression of cancers, including lung carcinoma, require several key steps including alteration in the control of cell proliferation, survival, metastasis, and evasion of apoptosis. In the present study, we found that overexpression of KLF5 promotes while knockdown of its expression inhibits cell proliferation in two cell lines derived from lung carcinoma. Previous studies have shown that KLF5 overexpression was sufficient to induce the expression of cell cycle genes, including Cyclin A, Cdt1, and E2F3 in breast cancer cells [21]. Besides, mediates Cyclin D1 expression and cell proliferation via interaction with c-Jun in vascular smooth muscle cells [22]. Therefore, our results together with these reports demonstrate that KLF5 may play an oncogenic role to promote cell proliferation and tumor development $[19,20]$. However, recent studies also suggest that expression of KLF5 is severely lost in human esophageal squamous cell cancer (ESCC) [23,24]. Loss of transcription factor KLF5 in the context of p53 ablation drives invasive progression of human squamous cell cancer [23]. Restoring KLF5 in ESCC cells activates the c-Jun N-terminal kinase pathway leading to apoptosis and reduced cell survival [24]. Although the reason for this inconsistency remains unclear, the role of KLF5 in the cell proliferation might be cell or tissue-specific, which may dependent on cellular context or different downstream target genes.

At the molecular level, our results further identified Sox 4 as a novel target gene of KLF5. Sox4, a member of SOX (Sry-box) family known, is initially implied in the development of the cardiac outflow tract, pro-B cell expansion and the central nervous system $[25,26]$. Subsequent studies reveal that Sox4 expression is positively correlated with progression of several human cancer types, suggesting that its overexpression might be a useful prognostic marker for cancers [27]. For instance, Sox4 induces epithelial-mesenchymal transition and contributes to breast cancer progression, by controlling Ezh2 expression and epigenetic reprogramming $[28,29]$. Besides, Sox4 interacts with p53 and inhibits p53mediated apoptosis in hepatocellular carcinoma [30]. Moreover, recent reports link the Sox4 expression to the development of small cell lung cancer, suggesting that it could also be a driver oncogene in lung carcinoma [31]. However, the molecular determinants of its expression remain largely unexplored. Therefore, our study also adds a novel mechanism for the dys-regulated Sox 4 expression in human cancers.

In sum, we have defined a novel role for KLF5 in lung carcinoma, an extremely common cancer worldwide with a particularly poor prognosis. Importantly, KLF5 overexpression promotes while its depletion represses cell proliferation, which is mediated by its upregulation of Sox4. Future investigations will be directed toward fully defining the KLF5/ 
Li et al.: KLF5 Promotes Lung Tumorigenesis

Sox4 regulatory axis in vivo to better delineate the molecular mechanisms underlying the pathogenesis of lung cancer.

\section{Acknowledgements}

This work is supported by Natural Science Foundation of China grant 81071664 (to Q.L.), Natural Science Foundation of China grant 81272714 (to Q.L.), Shanghai Pujiang Programe 13PJD025 (to Q.L.)

\section{References}

1 Katlic MR, Facktor MA, Berry SA, McKinley KE, Bothe A Jr, Steele GD Jr: ProvenCare lung cancer: a multiinstitutional improvement collaborative. CA Cancer J Clin 2011;61:382-396.

-2 Ramalingam SS, Owonikoko TK, Khuri FR: Lung cancer: New biological insights and recent therapeutic advances. CA Cancer J Clin 2011;61:91-112.

3 Li X, Wang J, Xu Z, Ahmad A, Li E, Wang Y, Qin S, Wang Q: Expression of sox2 and oct4 and their clinical significance in human non-small-cell lung cancer. Int J Mol Sci 2012;13:7663-7675.

4 Wen C, Dehnel T: China wrestles with lung cancer. Lancet Oncol 2011;12:15.

5 Kaczynski J, Cook T, Urrutia R: Sp1- and Krüppel-like transcription factors. Genome Biol 2003;4:206.

6 Wieschaus E, Nusslein-Volhard C, Kluding H: Krüppel, a gene whose activity is required early in the zygotic genome for normal embryonic segmentation. Dev Biol 1984;104:172-186.

7 McConnell BB, Yang VW: Mammalian Krüppel-like factors in health and diseases. Physiol Rev 2010;90:1337-1381.

8 Bieker JJ: Krüppel-like factors: three fingers in many pies. J Biol Chem 2001;276:34355-34358.

-9 Pearson R, Fleetwood J, Eaton S, Crossley M, Bao S: Krüppel-like transcription factors: a functional family. Int J Biochem Cell Biol 2008;40:1996-2001.

10 Lomberk G, Urrutia R: The family feud: Turning off Sp1 by Sp1-like KLF proteins. Biochem J 2005;392:111.

11 O'Grady E, Mulcahy H, Adams C, Morrissey JP, O'Gara F: Manipulation of host Krüppel-like factor (KLF) function by exotoxins from diverse bacterial pathogens. Nat Rev Microbiol 2007;5:337-341.

12 Ghaleb AM, Nandan MO, Chanchevalap S, Dalton WB, Hisamuddin IM, Yang VW: Krüppel-like factors 4 and 5: the yin and yang regulators of cellular proliferation. Cell Res 2005;15:92-96.

13 Wei D, Gong W, Kanai M, Schlunk C, Wang L, Yao JC, Wu TT, Huang S, Xie K: Drastic down-regulation of Krüppel-like factor 4 expression is critical in human gastric cancer development and progression. Cancer Res 2005;65:2746-2754.

14 Wei D, Kanai M, Jia Z, Le X, Xie K. Krüppel-like factor 4 induces p27Kip1 expression in and suppresses the growth and metastasis of human pancreatic cancer cells. Cancer Res 2008;68:4631-4639.

15 Lahiri SK, Zhao J: Krüppel-like factor 8 emerges as an important regulator of cancer. Am J Transl Res 2012;4:357-363.

-16 Watanabe N, Kurabayashi M, Shimomura Y, Kawai-Kowase K, Hoshino Y, Manabe I, Watanabe M, Aikawa M, Kuro-o M, Suzuki T, Yazaki Y, Nagai R: BTEB2, a Krüppel-like transcription factor, regulates expression of the SMemb/Nonmuscle myosin heavy chain B (SMemb/NMHC-B) gene. Circ Res 1999;85:182-191.

17 Shindo T, Manabe I, Fukushima Y, Tobe K, Aizawa K, Miyamoto S, Kawai-Kowase K, Moriyama N, Imai Y, Kawakami H, Nishimatsu H, Ishikawa T, Suzuki T, Morita H, Maemura K, Sata M, Hirata Y, Komukai M, Kagechika H, Kadowaki T, Kurabayashi M, Nagai R: Krüppel-like zinc-finger transcription factor KLF5/ BTEB2 is a target for angiotensin II signaling and an essential regulator of cardiovascular remodeling. Nat Med 2002;8:856-863.

18 Oishi Y, Manabe I, Tobe K, Tsushima K, Shindo T, Fujiu K, Nishimura G, Maemura K, Yamauchi T, Kubota N, Suzuki R, Kitamura T, Akira S, Kadowaki T, Nagai R: Krüppel-like transcription factor KLF5 is a key regulator of adipocyte differentiation. Cell Metab 2005;1:27-39. 
Li et al.: KLF5 Promotes Lung Tumorigenesis

19 Noto JM, Khizanishvili T, Chaturvedi R, Piazuelo MB, Romero-Gallo J, Delgado AG, Khurana SS, Sierra JC, Krishna US, Suarez G, Powell AE, Goldenring JR, Coffey RJ, Yang VW, Correa P, Mills JC, Wilson KT, Peek RM Jr: Helicobacter pylori promotes the expression of Krüppel-like factor 5, a mediator of carcinogenesis, in vitro and in vivo. PLoS One 2013;8:e54344.

20 Mori A, Moser C, Lang SA, Hackl C, Gottfried E, Kreutz M, Schlitt HJ, Geissler EK, Stoeltzing O: Up-regulation of Krüppel-like factor 5 in pancreatic cancer is promoted by interleukin-1beta signaling and hypoxiainducible factor-1alpha. Mol Cancer Res 2009;7:1390-1398.

21 Liu R, Zhou Z, Zhao D, Chen C: The induction of KLF5 transcription factor by progesterone contributes to progesterone-induced breast cancer cell proliferation and dedifferentiation. Mol Endocrinol 2011;25:11371144.

22 Liu Y, Wen JK, Dong LH, Zheng B, Han M: Krüppel-like factor (KLF) 5 mediates cyclin D1 expression and cell proliferation via interaction with c-Jun in Ang II-induced VSMCs. Acta Pharmacol Sin 2010;31:10-18.

23 Nakajima Y, Akaogi K, Suzuki T, Osakabe A, Yamaguchi C, Sunahara N, Ishida J, Kako K, Ogawa S, Fujimura T, Homma Y, Fukamizu A, Murayama A, Kimura K, Inoue S, Yanagisawa J: Estrogen regulates tumor growth through a nonclassical pathway that includes the transcription factors ERb and KLF5. Sci Signal 2011;4:ra22.

24 Tarapore RS, Yang Y, Katz JP: Restoring KLF5 in esophageal squamous cell cancer cells activates the JNK pathway leading to apoptosis and reduced cell survival. Neoplasia 2013;15:472-480.

25 Schilham MW, Oosterwegel MA, Moerer P, Ya J, de Boer PA, van de Wetering M, Verbeek S, Lamers WH, Kruisbeek AM, Cumano A, Clevers H: Defects in cardiac outflow tract formation and pro-B-lymphocyte expansion in mice lacking Sox-4. Nature 1996;380:711-714.

26 Cheung M, bu-Elmagd M, Clevers H, Scotting PJ: Roles of Sox4 in central nervous system development. Brain Res Mol Brain Res 2000;79:180-191.

27 Vervoort SJ, van Boxtel R, Coffer PJ: The role of SRY-related HMG box transcription factor 4 (SOX4) in tumorigenesis and metastasis: friend or foe? Oncogene 2013;32:3397-3409.

28 Zhang J, Liang Q Lei Y, Yao M, Li L, Gao X, Feng J, Zhang Y, Gao H, Liu DX, Lu J, Huang B: SOX4 induces epithelial-mesenchymal transition and contributes to breast cancer progression. Cancer Res 2012;72:4597-4608.

29 Tiwari N, Tiwari VK, Waldmeier L, Balwierz PJ, Arnold P, Pachkov M, Meyer-Schaller N, Schübeler D, van Nimwegen E, Christofori G: Sox4 is a master regulator of epithelial-mesenchymal transition by controlling Ezh2 expression and epigenetic reprogramming. Cancer Cell 2013;23:768-783.

-30 Hur W, Rhim H, Jung CK, Kim JD, Bae SH, Jang JW, Yang JM, Oh ST, Kim DG, Wang HJ, Lee SB, Yoon SK: SOX4 overexpression regulates the p53-mediated apoptosis in hepatocellular carcinoma: clinical implication and functional analysis in vitro. Carcinogenesis 2010;31:1298-1307.

- 31 Castillo SD, Matheu A, Mariani N, Carretero J, Lopez-Rios F, Lovell-Badge R, Sanchez-Cespedes M: Novel transcriptional targets of the SRY-HMG box transcription factor SOX4 link its expression to the development of small cell lung cancer. Cancer Res 2012;72:176-186. 